Sección uno: Ensayo

Radiografía de la innovación educativa en el Siglo XXI

\title{
Una app gamificada para incrementar la motivación hacia el aprendizaje de idiomas ${ }^{1}$
}

A gamified App to enhance motivation towards language learning

\author{
Anke Berns, \\ Universidad de Cádiz \\ anke.berns@uca.es \\ Manuel Palomo-Duarte \\ Universidad de Cádiz \\ manuel.palomo@uca.es
}

\section{Resumen}

La creciente expansión de las tecnologías móviles ha creado nuevas oportunidades y desafíos para la enseñanza y el aprendizaje de idiomas. No obstante, a pesar de que en la última década se hayan desarrollado numerosas aplicaciones comerciales y no comerciales (Apps) para fomentar el aprendizaje de idiomas, pocas explotan el potencial que ofrecen hoy en día los smartphones o tabletas para crear nuevos entornos de aprendizaje. En cambio, la mayoría siguen centrándose en un aprendizaje formal e individual, sin aprovechar las posibilidades que brindan los actuales dispositivos móviles para incrementar la interacción y comunicación entre varios usuarios. De ahí nuestro propósito de desarrollar una app que no solo permita la interacción entre sus usuarios proponiendo un enfoque basado en el aprendizaje comunitario sino que además explote el potencial de la gamificación para incrementar la motivación del alumnado. Para ello se ha implementado una app gamificada que permite al usuario introducir nuevo contenido (acorde a sus necesidades de aprendizaje) además de evaluar y reportar el contenido creado por otros usuarios y de recibir feedback regular sobre su propio proceso de aprendizaje.

${ }^{1}$ Recibido: 30/11/2019 Evaluado: 10/12/2019 Aceptado: 09/02/2020 
Palabras clave: smartphones, aprendizaje ubicuo, aprendizaje comunitario.

\begin{abstract}
The increasing expansion of mobile technologies (smartphones, tablets, etc.) has created new opportunities and challenges for the teaching and learning of languages. However, despite the numerous commercial and non-commercial apps that have been developed in the last decade, very few applications (apps), explore the potential that current smartphones and tablets offer to create new learning environments. In fact, most of the apps still focus its interest on supporting form-focused and individual learning rather than exploiting the potential of current mobile devices to enhance students' interaction and communication amongst each other. Therefore, the present study aims at developing a gamified app that not only might let its users to interact amongst each other providing a community-driven learning approach but also exploits the potential of gamification to enhance students' motivation. To this end we have implemented a system that allows its users to add new content based on their learning needs, evaluate and report the learning content that is gradually created by other users.
\end{abstract}

Keywords: smartphones, ubiquitous learning, community driven learning.

\title{
Introducción
}

El desarrollo y uso de apps de idiomas está cada vez más extendido, debido a que la mayoría de los actuales dispositivos móviles (smartphones y tabletas) ofrecen nuevas posibilidades para enriquecer los procesos de enseñanza-aprendizaje (Berns et al., 2017; Kumar y Mohite, 2018). En este sentido no es de extrañar que en los últimos años haya habido numerosos intentos, por parte de editoriales, docentes e investigadores, de explorar su potencial educativo y motivador. Prueba de ese creciente interés son, entre otros, los resultados de varias revisiones de la literatura, realizados entre el 2006 y 2018 (Burston 2013; 2014a y b; 2015; Chinnery, 2006; Duman, Orhon y Gedik, 2015; Godwin-Jones, 2011; Heil, Wu, Lee y Schmidt, 2016; Kukulska-Hulme, 2008; Kukulska-Hulme y Viberg, 2018). Si bien estas revisiones dan fe de la enorme cantidad de apps que se diseñaron durante la última década, el análisis también demuestra que pocas de las apps que se encuentran actualmente disponibles en plataformas como Google Play y App Store o en la literatura académica que se centra en el análisis de MALL proporcionan un enfoque más allá de una implementación digital de ejercicios clásicos de vocabulario, gramática, etc. Tanto es así que la gran mayoría de las apps siguen centrándose en el aprendizaje formal e individual del estudiante, dejando de lado las múltiples posibilidades que los actuales dispositivos móviles ofrecen para fomentar la interacción, comunicación o, incluso, el aprendizaje comunitario entre varios usuarios (Berns, Palomo-Duarte, Dodero e Isla-Montes, 2016; Berns et al., 2017).

La app (Guess It! LanguageTrainer) que se presenta en este estudio fue diseñada atendiendo precisamente a la necesidad de explotar el potencial de los dispositivos móviles para desarrollar entornos dinámicos de aprendizaje, permitiendo al usuario adquirir tanto nuevo 
input lingüístico (vocabulario, gramática, estructuras lingüísticas) como aplicarlo en la interacción y negociación con otros usuarios.

\section{Metodología y marco teórico}

Guess It! Language Trainer, que se desarrolló para el presente proyecto, parte, por un lado, del marco teórico de aprendizaje móvil propuesto por Sharples, Taylor y Vavoula, 2016 y, por otro, del marco teórico de la autodeterminación y motivación intrínseca y extrínseca de Ryan y Deco, 2000. En su estudio los Sharples et al., (2016) subrayan que el valor añadido del aprendizaje móvil reside no solo en las numerosas posibilidades que este brinda en términos de ubicuidad y opciones para permitir la interacción entre múltiples usuarios sino, al mismo tiempo, en las posibilidades que brindan para el aprendizaje colaborativo. Es por esto que entiendan el aprendizaje móvil como un proceso en el cual se adquieren conocimientos, gracias a la interacción que se produce entre las tecnologías y sus usuarios. De acuerdo con los aspectos mencionados, estos autores proponen un marco teórico que parte de la teoría de la actividad de Engeström (1987), quien analiza las interdependencias entre el aprendizaje y el uso de las tecnologías. El modelo que Engeström propone consta de cinco factores (sujeto, objeto, contexto, herramientas y comunicación), que a su vez son analizados a nivel tecnológico y a nivel semiótico.El nivel tecnológico describe el aprendizaje como un compromiso con la tecnología en el cual dispositivos como los teléfonos móviles actúan como agentes interactivos que ayudan a sus usuarios a adquirir nuevos conocimientos, a comunicarse entre ellos, a compartir y negociar contenidos y significados y, finalmente a reflexionar sobre los contenidos proporcionados. En cambio, el nivel semiótico describe el aprendizaje como un sistema cognitivo en el cual las acciones de los aprendientes son mediadas por medio de herramientas culturales y símbolos (Sharples et al., 2016). Junto al marco teórico de Sharples et al., 2016 fueron importantes las reflexiones deRyan y Deco, 2000 con respecto a la autodeterminación y motivación (tanto intrínseca como extrínseca) consideradas como factores claves para fomentar el aprendizaje. Así los autores insisten en su estudio en la importancia de fomentar en determinados contextos (estudios, etc.) que a menudo requieren la realización de actividades y tareas poco motivadoras aquellos factores que puedan convertir lo "no deseado" en motivador. Como aspectos claves en este proceso destacan promover tanto la autodeterminación por parte del estudiante como factores que favorecen la motivación del estudiante (actividades que suponen un desafío, permiten satisfacer el deseo natural del ser humano de aprender, facilitan conocimientos útiles).

En línea con lo anteriormente dicho uno de los aspectos claves para el diseño de Guess It! Language Trainer fue la implementación de herramientas capaces de contribuir al aprendizaje del usuario mediante la construcción, evaluación y participación activa de la comunidad de usuarios (Bellotti, Kapralos, Lee, Moreno-Ger y Berta, 2013). Esto implicaba que la app tenía que cumplir una serie de requisitos entre los cuales cabe destacar los siguientes: uno, el contenido tenía que partir del entorno, de los conocimientos e intereses de los usuarios destinatarios (en nuestro caso estudiantes del nivel A1 de Alemán). Dos, los contenidos de aprendizaje, tenían que construirse a partir de un conocimiento previamente validado (Sharples et al., 2016). Tres, la evaluación tenía que de dar respuesta a las necesidades de los respectivos usuarios de recibir un feedback constante e individualizado durante todo su proceso de aprendizaje. Y cuatro, que la app tenía que motivar a los usuarios a participar activamente en la creación de los contenidos y, por lo tanto, en la co-construcción 
de los conocimientos de la lengua meta (Palomo-Duarte,Berns, Cejas, Dodero, CaballeroHernández y Ruiz-Rube, 2018).

En el contexto descrito, GuessIt! LanguageTrainer pretende ofrecer un entorno de aprendizaje interactivo y dinámico, capaz de permitir a sus usuarios tanto recibir un input lingüístico muy variado en lengua meta (en este caso del alemán) como de poder usar este input posteriormente para crear a partir de él nuevos contenidos de aprendizaje y compartir estos con el resto de la comunidad de usuarios. Al involucrar a los usuarios directamente en el proceso del diseño queremos dar respuesta a la creciente demanda de proporcionar un aprendizaje centrado en el estudiante y sus necesidades como usuario principal del producto final (Berns et al., 2016; Kennedy y Levy, 2009; Nelson y Oliver, 1999).

\section{Diseño de la app}

\section{Diseño de los contenidos de la app}

En términos de contenido, Guess It! Language Trainer facilita a sus usuarios diferentes ejercicios, que hacen hincapié en la comprensión oral y lectora como también en la expresión escrita. Para ello, Guess It! Language Trainer parte de un juego basado en adivinar palabras (vocabulario) a partir de un determinado contexto que viene facilitado por el propio sistema de la app mediante enunciados escritos (véase Figura 1).

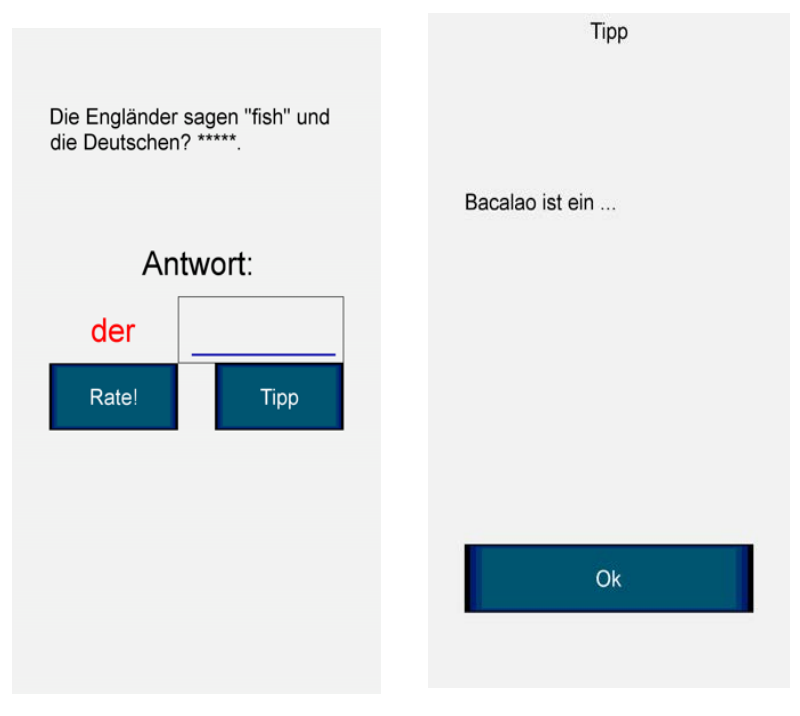

Figura 1. Ejemplo de una descripción de palabra y una pista para el usuario.

El contenido se diseñó a partir de los descriptores que facilita el Marco Común Europeo para las Lenguas (MCERL) para el nivel A1 (Ministerio de Educación, Cultura y Deporte, 2002). Posteriormente y en función de la secuenciación e impartición de los diferentes contenidos lingüísticos del curso objeto de este estudio (Alemán. Idioma Moderno I), la docente encargada de la asignatura procedió a dividir el temario en 4 niveles y 14 temáticas diferentes (tiempo libre, deportes, profesiones, lugares, comidas, bebidas, etc.). Aún así, el sistema se 
diseñó de tal modo que, dependiendo de las necesidades de otros docentes y usuarios destinatarios, fuera fácil la modificación e implementación de sus contenidos, niveles de dificultad e, incluso, de la lengua meta.

Así mismo, aunque Guess It! Language Trainer proporciona a sus usuarios un corpus inicial de frases y palabras contextualizadas, la app está configurada de tal modo que su contenido va creciendo y refinándose conforme que los usuarios vayan jugando e interactuando con la app. El hecho de que la interacción de los usuarios se basa no sólo en acertar palabras (a partir de diferentes contextos) sino,además, en evaluar y reportar las palabras y frases jugadas, permite, por un lado, crear un entorno de aprendizaje dinámico y altamente interactivo y, por otro, involucrar a los propios usuarios (estudiantes) activamente en el diseño del contenido de la app (Gikandi, Morrow y Davis, 2011; Taras, 2002; Van Zundert, Sluijsmans y van Merriënboer, 2010) y la co-construcción de sus conocimientos de lengua meta(Palomo-Duarte,Berns, Cejas, Dodero, Caballero-Hernández y Ruiz-Rube, 2018).

\section{Arquitectura del sistema}

En cuanto a la arquitectura del sistema, Guess It! Language Trainer se basa en una arquitectura cliente-servidor (Palomo-Duarte, Berns, Cejas, Dodero, Caballero-Hernández y Ruiz-Rube, 2018), en la cual el servidor coordina múltiples smartphones (o tabletas).Gracias a la conexión del servidor con la red, el sistema permite identificar la interacción de cada usuario con la app. Con respecto a la dinámica de la app, los usuarios deben primero completar una serie de oraciones introduciendo la palabra que falta en cada una de ellas. Dichas oraciones vienen proporcionadas por un algoritmo de selección, que fue implementado en la app y el cual se activa en el momento en el que los usuarios empiezan a jugar una partida. A lo largo de cada partida los usuarios deben primero completar las oraciones proporcionadas y luego evaluar su calidad en función de una serie de factores: si la oración y palabra contextualizada son correctos (en términos de gramática y contenido), si son precisos (si se entiende o, si, por lo contrario, resulte ambigua) o, por lo contrario, difíciles de acertar o, incluso, ofensivos.

Una vez que un usuario haya jugado y evaluado un determinado número de frases y palabras contextualizadas, el sistema le permite crear sus propias oraciones para contextualizar una o más palabras que le va proporcionando el propio algoritmo de la app. Para ello le provee, por cada 20 frases/palabras jugadas, con una palabra (sustantivo, verbo o adjetivo) de la base de conocimiento para la cual deberá, a continuación, crear una nueva oración ejemplo (véase Figura 2). De este modo el sistema invita a los usuarios a crear sus propios materiales de aprendizaje, permitiéndoles adaptar los contenidos a sus necesidades como aprendientes. 


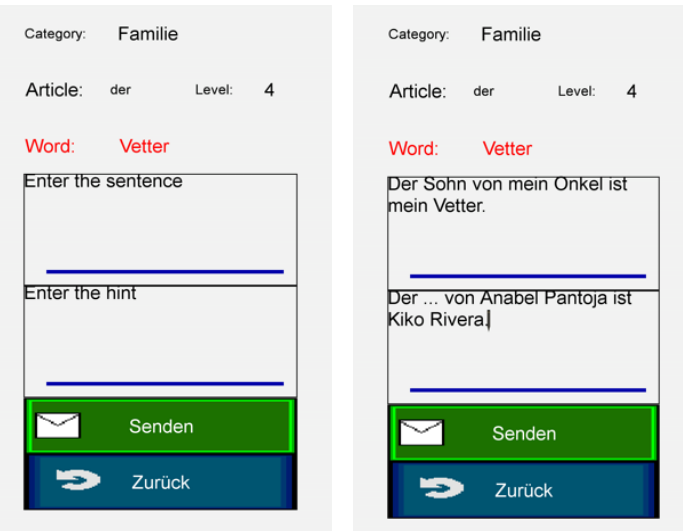

Figura 2. Ejemplo de una palabra descrita por un estudiante del curso.

Una vez que el usuario haya creado e introducido su propia oración ejemplo, esta entra en la base de conocimiento y se pone a disposición de los demás usuarios, que, a su vez, con sus posteriores evaluaciones y reportes determinarán la frecuencia con la que cada oración ejemplo aparecerá en las siguientes partidas.

Como resultado de este proceso, el servidor contendrá diversas oraciones de ejemplo por cada palabra y varias evaluaciones para cada una de las oraciones almacenadas. Esto permite que aquellas oraciones ejemplo que obtuvieron, en un determinado momento, evaluaciones más altas por parte de los usuarios, serán seleccionadas por el algoritmo con mayor frecuencia, mientras que aquellas que obtuvieron una valoración más baja, serán seleccionadas con menor frecuencia. No obstante, ninguna de las oraciones ejemplo serán nunca eliminadas definitivamente para así poder ser re-evaluadas, en otro momento, por los mismos usuarios (Berns et al., 2016).

Este procedimiento permite que en caso de que una oración ejemplo -aún siendo lingüísticamente correcta- haya obtenido alguna vez una valoración baja pueda ser reevaluada en otro momento de manera más objetiva y justa por otros usuarios con un mejor conocimiento de la lengua meta y, por lo tanto, con otros criterios de evaluación.

\section{Implementación de elementos de gamificación}

Con el fin de incrementar el potencial motivador de Guess It! Language Trainer, se usaron además para su diseño varios elementos de gamificación. La gamificación se entiende como un proceso en el cual se recurre a técnicas, elementos y dinámicas lúdicos con el objetivo de convertir una actividad, que a priori puede ser concebida como “aburrida” (estudiar, trabajar, etc.), en una actividad atractiva, dinámica y motivadora para sus participantes (Monguillot Hernando, González Arévalo, Zurita Mon, AlmirallBatet y GuitertCatasús, 2015;Carpena, Cataldi y Muñiz, 2012; Pérez-Pueyo y Hortigüela, 2020). Entre las técnicas más frecuentemente usadas cabe señalar el uso de niveles, feedback, puntos y rankings (Faiella y Ricciardi, 2015; Hasegawa, 2015; Lee y Hammer, 2011; Nacke y Deterding, 2017; Subhash y Cudney, 2018), técnicas que se han usado también, como se ilustra a continuación, para el diseño de Guess It! Language Trainer. 
Niveles: se ofrecen un total de cuatro niveles, según el grado de dificultad que representa cada una de las oraciones ejemplo que contiene la base de conocimiento.

Retroalimentación: se ofrecen dos tipos de retroalimentación (llamados a partir de aquí feedback): un feedback inmediato después de cada oración/ palabra jugada y un feedback más explícito, al final de cada partida. Mientras el feedback inmediato consiste en indicar al usuario si acertó o no la respuesta (facilitando además la respuesta correcta después del tercer intento fallido), el feedback explícito ofrece al usuario un resumen detallado de los resultados de una partida completa, incluyendo las oraciones tanto acertadas como no acertadas.

Puntos/ Rankings: además del feedback inmediato y explícito, se ofrecen al estudiante una serie de estadísticas de rendimiento junto a los puntos y reportes obtenidos a lo largo de las diferentes partidas. En términos de rankings se indica a cada usuario la temática, tanto en la que más como en la que menos oraciones y palabras acertó. Asimismo, se indican tanto el número total de niveles jugados como los niveles más jugados. En cuanto a los puntos obtenidos, a lo largo de las diferentes partidas, estas se representan en forma de porcentaje de acierto de las descripciones jugadas (rendimiento) y del total de descripciones jugadas (esfuerzo). Asimismo, se incluye la ratio de acierto y evaluaciones que han tenido las oraciones ejemplo introducidas por el mismo jugador. Esta ratio se calcula en base a las evaluaciones que una determinada oración ejemplo ha obtenido por otros usuarios.

A pesar del valor que atribuyen numerosas investigaciones a la gamificación para incrementar la motivación y el aprendizaje del alumnado existen también investigaciones que advierten de su peligro. En este sentido Pérez López (2019) junto a Pérez Pueyo y Hortigüela Alcalá (2020)indican en sus estudios que el uso de la gamificación de por si no puede ser un garante suficiente para lograr una mejora de la motivación y del aprendizaje a largo plazo sino más bien a corto plazo. Las razones que alegan son el hecho de que si bien la gamificación puede tener un efecto positivo en la motivación y el aprendizaje del alumnado, este impacto positivo se debe muchas veces a su carácter novedoso, logrando un cambio momentáneo, pero no necesariamente duradero (Ermi y Mäyrä, 2005). De ahí que insisten en la importancia de hacer un uso más consciente de la gamificación y de su potencial para mejorar los procesos de enseñanza-aprendizaje sin dejar de lado la revisión de los planteamientos pedagógicos que se hallan detrás de nuestra práctica docente y que, a menudo, son el verdadero causante del fracaso educativo y de la desmotivación del alumnado (SeinEchaluce, M.L. Fidalgo-Blanco y Alves, 2017; Pérez Pueyo y Hortigüela Alcalá, 2020).

\section{Experiencia}

\section{Participantes e instrumentos}

GuessIt! Language Trainer ha sido puesta en práctica con más de 100 estudiantes de alemán de la Universidad de Cádiz. La experiencia se realizó con estudiantes, que en el momento del experimento estuvieron cursando la asignatura de Idioma Moderno I (nivel A1.2 del MCERL). Uno de los principales objetivos de la asignatura consiste en el desarrollo de la comprensión/ expresión oral y escrita. Dado que en el aula se suele contar cada curso académico con hasta 80 estudiantes por grupo, se hace necesario completar el aprendizaje 
dentro del aula con el aprendizaje autónomo efectivo fuera del aula de acuerdo al creditaje ECTS de la asignatura.

La experiencia, cuyos resultados presentamos, duró cuatro semanas. Durante ellas, los estudiantes fueron invitados primero a jugar los niveles 1 y 2 (primera semana), después el nivel 3 (segunda semana) y finalmente los niveles 1 a 4 (tercera y cuarta semana).

Antes de dar a los estudiantes acceso al contenido de la app quisimos analizar sus conocimientos lingüísticos previos a usar Guess It! Language Trainer a fin de medir, una vez terminada la experiencia, su impacto en el aprendizaje de los estudiantes. Por ello les pedimos participar primero en un pre-test, que contenía un total de 60 oraciones ejemplo, las cuales fueron aleatoriamente seleccionadas de la base de conocimiento de Guess It! Language Trainer (Tabla 1).

\begin{tabular}{|l|l|l|l|}
\hline Tema & Nivel & Oración & Palabra \\
\hline $\begin{array}{l}\text { Essen- } \\
\text { Trinken }\end{array}$ & 1 & $\begin{array}{l}\text { Rum, Bier und Whisky sind ... Das } \\
\text { Wortbeginntmit "A". }\end{array}$ & \\
\cline { 2 - 4 } & 2 & $\begin{array}{l}\text { EinGetränkmit "W". Rioja istein ... } \\
\text { ausSpanien. }\end{array}$ & \\
\cline { 2 - 5 } & 3 & $\begin{array}{l}\text { EinWortmit "D". IchtrinkeeinGlas, eine } \\
\text {.. odereineFlasche Bier. }\end{array}$ & \\
\cline { 2 - 5 } & 4 & $\begin{array}{l}\text { Wenn du ... hast, danntrinkst du } \\
\text { Wasserusw. Wortbeginntmit "D". }\end{array}$ & \\
\hline Wetter- & 1 & $\begin{array}{l}\text { EinAdjektivmit "h". Das Gegenteil von } \\
\text { "kalt" ist ... }\end{array}$ & \\
\cline { 2 - 5 } & 2 & $\begin{array}{l}\text { EinAdjektivmit "sch". Das Wetter ist ... } \\
\text { Das Gegenteil von "schön". }\end{array}$ & \\
\cline { 2 - 5 } & 3 & $\begin{array}{l}\text { In England ist das Wetter oft schlecht. } \\
\text { Was ist das Problem? Es ... viel. Das } \\
\text { Wortbeginntmit "r". }\end{array}$ & \\
\cline { 2 - 5 } & 4 & $\begin{array}{l}\text { EinWortmit "N". } \\
\text { AndalusienliegtimSüden von Spanien } \\
\text { und Galizienliegtim ... }\end{array}$ & \\
\cline { 2 - 5 } & & &
\end{tabular}

Tabla 1. Preguntas ejemplo del pre-test y post-test 2.

Una vez terminado el pre-test, se dio a los estudiantes acceso a la app familiarizándolos, durante una primera sesión introductoria y presencial, con la interfaz y las diferentes herramientas de la app como también con sus contenidos y objetivos didácticos. Después de esta primera sesión introductoria se les pidió usar la app como apoyo a su aprendizaje autónomo, fuera del aula. 
Tras jugar la primera semana el nivel 1, se procedió a realizar un primer post-test que constaba igualmente de un total de 60 oraciones ejemplo(abarcando un total de 15 temáticas diferentes, correspondientes al nivel A1 del MCERL: comidas-bebidas, el tiempo, deportes, profesiones, etc.), pero esta vez solamente centrado en el nivel 1. A este primer post-test siguieron un segundo (niveles 2 y 3 ) en la tercera semana del experimento y un tercer posttest (niveles 1 a 4) en la cuarta semana. Todos los tests fueron realizados durante las clases presenciales y contenían el mismo número y tipo de tareas (palabras a acertar a partir de una determinada frase ejemplo y crear otras frases ejemplo, a partir de una determinada palabra), todas ellas seleccionadas anteriormente de forma aleatoria de la base de conocimiento.

Lo que diferenciaba cada etapa de la experiencia (suponiendo un incremento de desafío para los estudiantes a lo largo de la experiencia) fue el número de frases ejemplo, que contenía inicialmente cada nivel y que iba aumentando, conforme que los estudiantes iban jugando e interactuando con la app. Así, mientras el post-test 1 (niveles 1 y 2) partía de un corpus léxico de un total de 223 frases ejemplo, seguido por el post-test 2 (nivel 3), que partía de unas 65 frases ejemplo, este corpus incrementó de forma notable a lo largo del experimento alcanzando en la tercera y cuarta semana del experimento un total de 826 frases ejemplo (niveles 1 a 4). Hubo por lo tanto un incremento del corpus inicial (282 frases ejemplos) con respecto al corpus final (826 frases ejemplo) de más de 544 frases ejemplo.

\section{Análisis y resultados}

\section{Análisis de los resultados de aprendizaje}

Un análisis comparativo de los resultados del pre-test (niveles 1-4) al tercer post-test (niveles 1-4) demuestra el impacto de Guess It! Language Trainer en el aprendizaje de los estudiantes. No solo mejoraron significativamente sus conocimientos de la lengua meta en términos de vocabulario y gramática sino también en cuanto a su competencia escrita. En este sentido se pudo observar una diferencia media en los resultados globales de 5.35 puntos en un rango de 0 a 10. Las mejoras intermedias también fueron significativas: 4.8 puntos del pre-test al primer post-test, y 5.7 del pre-test al segundo post-test.

En total los 150 estudiantes registrados en el sistema jugaron un total de 165.178 oraciones ejemplo. Estos resultados son especialmente notables teniendo en cuenta que la experiencia duró apenas cuatro semanas a lo largo de las cuales el contenido lingüístico, al que fueron expuestos, incrementó de manera significativa.

Los resultados de la experiencia demuestran que más del 85\% de las oraciones ejemplo (que jugaron los estudiantes) recibieron la máxima nota posible, y algo más del 11\% de las oraciones ejemplo recibieron 3 puntos sobre 4 . Por lo tanto, solo el 3\% de las oraciones ejemplo recibieron una nota media o baja. Teniendo en cuenta que el corpus inicial fue de 282 oraciones ejemplo introducidas por la docente de la asignatura y terminó teniendo un total de 850 oraciones ejemplo, los estudiantes introdujeron unas 568 oraciones ejemplo (un $66 \%$ del corpus final).

En cuanto a los reportes (oraciones ejemplo que algún jugador marcó como incorrectas), se puede observar que hubo un total de 11.000 oraciones ejemplo, que fueron reportadas. De 
estas algunas recibieron más de 100 reportes, lo cual parece un indicador claro de que probablemente sean incorrectas. Sin embargo, otras 395 oraciones ejemplo recibieron menos de 10 reportes (dato que hace pensar que los estudiantes que reportaron estas oraciones ejemplo lo hicieron de manera incorrecta, mostrando lagunas en sus conocimientos de la lengua meta). Esta hipótesis parece confirmarse también teniendo en cuenta que hubo estudiantes que reportaron incluso oraciones ejemplo que fueron introducidas por la propia docente de la asignatura.

\section{Valoración de la app por parte de los estudiantes}

A fin de valorar la aceptación de Guess It! Language Trainer, por parte de nuestros estudiantes, les pedimos rellenar un cuestionario al final de la experiencia. El cuestionario fue diseñado dentro del marco del Technology Acceptance Model (TAM), propuesto por (Liu, Chen, Sun, Wible y Kuo, 2010) y usando una escala Likert (muy de acuerdo=5; de acuerdo $=4$, normal=3; en desacuerdo=2; muy desacuerdo=1). Los aspectos que quisimos analizar fueron uno, la valoración por parte de los estudiantes con respecto al "Diseño de la app” (DAM) y el grado en que este correspondía a las necesidades de los usuarios; dos, la "Utilidad percibida" (UP) en cuanto al aprendizaje de diferentes aspectos lingüísticos (vocabulario, gramática, estructuras sintácticas, etc.); tres, la "Interacción percibida” (IP) al usar la app y al interactuar con otros usuarios y cuatro, la "Intención de uso de apps para móvil” (IUAM). Este último aspecto pretende analizar la actitud y aceptación del estudiante hacia el uso futuro (más allá de la experiencia realizada con Guess It! Language Trainer) de apps para el aprendizaje de idiomas (Tabla2).

\begin{tabular}{|l|}
\hline 1. Diseño de la app para móvil (DAM) \\
\hline 1.1. El contenido de la app es interesante. \\
\hline 1.2 El contenido de la app corresponde a mis necesidades de aprendizaje. \\
\hline 1.3 El feedback, proporcionado por la app, es muy útil. \\
\hline 1.4 En general, estoy satisfecho con el contenido y la calidad de la app. \\
\hline 2. Utilidad Percibida (UP) \\
\hline 2.1 Este tipo de apps es útil para mejorar mi competencia lingüística. \\
\hline 2.2. Este tipo de apps es útil para mejorar mi expresión escrita. \\
\hline 2.3 Este tipo de apps es útil para mejorar mi comprensión lectora. \\
\hline
\end{tabular}




\begin{tabular}{|l|}
\hline 2.4 Este tipo de apps es útil para aprender vocabulario. \\
\hline 2.5 Este tipo de apps es útil para comunicarse con más fluidez en alemán. \\
\hline 3. Interacción Percibida (IU) \\
\hline $\begin{array}{l}\text { 3.1 En general, creo que las apps para teléfonos móviles y combinadas con juegos, } \\
\text { ofrecen buenas oportunidades para practicar una lengua extranjera. }\end{array}$ \\
\hline 4. Intención de usar app para móvil (IUAM) \\
\hline $\begin{array}{l}\text { 4.1 Pretendo usar este tipo de apps más a menudo para mejorar mi nivel de alemán } \\
\text { (o el de otros idiomas). }\end{array}$ \\
\hline 4.2 Usaría este tipo de apps para mejorar mi expresión escrita. \\
\hline 4.3 Usaría este tipo de apps para más actividades de aprendizaje fuera de clase.
\end{tabular}

Tabla 2. Cuestionario TAM que se pasó a los participantes al final de la experiencia.

En cuanto a la valoración que hicieron los 104 estudiantes que participaron finalmente en el cuestionario, cabe destacar que 76 estudiantes valoraron el diseño de la app y, en concreto, el contenido muy positivamente (5 sobre 5), destacando, ante todo, su aspecto motivador. Asimismo, 51 de los estudiantes confirmaron que el contenido de la app corresponde a sus necesidades de aprendizaje (valorando su contenido entre 4 y 5 sobre 5), seguido por 60 que afirmaron que el feedback proporcionado durante cada partida, les fue muy útil (5 sobre 5) a la hora de progresar en su aprendizaje (Tabla 2, preguntas 1.1-1.3). El feedback se dividía tanto en feedback inmediato (valorando con verdadero o falso las respuestas dadas) como en un feedback más explícito, que se ofreció al final de cada palabra (no) acertada. El feedback explícito tenía como objetivo por un lado indicar al estudiante los errores cometidos y, por otro, facilitar al estudiante posibles respuestas y así poder mejorar sus resultados en partidas posteriores.Aunque la valoración en cuanto a satisfacción global con la app (Tabla 2, pregunta 1.4) sufre una valoración ligeramente más baja con respecto a las valoraciones anteriores (solo 41 estudiantes estuvieron totalmente satisfechos), estos resultados se deben (según lo que confirmaron varios estudiantes en entrevistas personales, al terminar la experiencia) a una serie de errores técnicos de la versión Beta que se usó para esta primera experiencia.

En cuanto al segundo aspecto (Utilidad Percibida) la aceptación positiva de la app, por parte de los estudiantes, viene también confirmada con respecto a los resultados de aprendizaje logrado. Así 84 de los estudiantes confirmaron que Guess It! Language Trainer les ayudó a aprender nuevo vocabulario, seguido por 83 que confirman haber mejorado su expresión 
escrita, 69 su comprensión lectora y 61 su competencia lingüística en general (Tabla 2, preguntas 2.1-2.5).

Este resultado viene además reforzado por la valoración del tercer y cuarto aspecto (Interacción percibida e Intención de usar app para móvil). Así que 79 estudiantes perciben apps combinadas con elementos de gamificación como herramientas muy útiles para el aprendizaje (Tabla 2, pregunta 3.1). Y, finalmente, 69 estudiantes destacan el potencial de la Guess It! Language Trainer para mejorar la expresión escrita, de los cuales 63 confirman además la intención de usar apps (como la que se usó durante la experiencia descrita aquí) más a menudo para facilitar el aprendizaje autónomo (Tabla 2, preguntas 4.1-4.2).

Solo 17 de los 104 estudiantes entrevistados se mostraron algo escépticos con respecto a usar apps para su aprendizaje de idiomas. Una de las razones que indicaron fue que muchos de ellos tenían un iPhone, el cual era incompatible con el sistema Android, sistema que se usó para diseñar Guess It! Language Trainer. Esto obligó a varios estudiantes a pedir los dispositivos móviles a familiares o amigos, lo cual limitaba su flexibilidad a la hora de usar la app. Otro factor que incidió, en algunos casos, en una aceptación menor de la app fueron varios errores en el sistema, debido a que en el momento del experimento disponían solamente de la versión Beta de la app. No obstante, los errores detectados en su momento (principalmente debidos a las distintas versiones del sistema Android instalado en los smartphones de los estudiantes) pudieron ser subsanados gracias al feedback de los participantes, permitiéndonos revisar y mejorar la app, que se usó durante la primera experiencia.

\section{Discusión y conclusiones}

Las tecnologías móviles han cambiado no solo nuestra forma de relacionar y comunicarnos, sino también nuestra forma de aprender. A lo largo de este estudio hemos descrito una experiencia piloto que fue desarrollada con una app gamificada, llamada Guess It! Language Trainer, que implementa una versión digital del clásico juego de adivinanza que consiste en adivinar palabras a partir de un determinado contexto. Al diseñar una app gamificada quisimos aprovechar el potencial motivador de dos de las herramientas que han ido ganando en estos últimos años en popularidad entre nuestros estudiantes: los smartphones y los videojuegos colaborativos. De ahí que diseñamos una app para smartphones que no solo permite la interacción con el contenido de la propia app y sus usuarios, sino que además está enriquecida con elementos de gamificación.

Los resultados del presente estudio demuestran que ambos factores tuvieron un impacto muy positivo tanto en el aprendizaje como en la motivación de los estudiantes. En cuanto a la configuración del sistema cabe destacar que la app permite la fácil adaptación a otros idiomas y/o contenidos, de modo que es fácilmente aplicable a la enseñanza de otros idiomas y niveles.

El rendimiento de la experiencia se evaluó en primer lugar usando un pre-test y tres posttests. Los resultados fueron muy positivos, mostrando una mejora significativa en el aprendizaje de los participantes en la experiencia. Además, los estudiantes rellenaron un 
cuestionario TAM sobre diversos aspectos de la app en el que los elementos de gamificación incluidos en ella fueron valorados muy positivamente.

A pesar de la valoración altamente positiva por parte de los estudiantes, la experiencia proporcionó a los autores una serie de sugerencias muy valiosas en cuanto a posibles mejoras de algunos aspectos técnicos y didácticos para convertir la app, en un futuro, en una herramienta de aprendizaje aún más eficaz. Entre las propuestas de mejora cabe destacar, entre otros, la implementación de un sistema de monitorización que, al detectar estudiantes con bajo rendimiento, sea capaz de activar mecanismos para proporcionar un feedback y apoyo más individualizado durante el proceso de aprendizaje. Asimismo, los autores están trabajando en la integración de un Lenguaje Específico de Dominio (LED) en el sistema. Dicha integración tiene como objetivo facilitarle al docente de idiomas la obtención de informes personalizados de la actividad de sus estudiantes a fin de mejorar el proceso de monitorización del proceso de aprendizaje.

\section{Reconocimientos}

El proyecto fue desarrollado dentro del proyecto VISAIGLE (TIN2017-85797-R) que está financiado por la Agencia Estatal de Investigación.

\section{Referencias}

Bellotti, F., Kapralos, B., Lee, K., Moreno-Ger, P. y Berta, R. (2013). Assessment in and of serious games: An overview. Advances in Human-Computer Interaction, 1, pp.1-11. doi:10.1155/2013/136864.

Berns, A. y Palomo-Duarte, M. (2015). Supporting Foreign-Language Learning through a gamified app. En R. Hernández y T. Rankins (Eds.), Third-level Education and Second Language Learning: Promoting Self directed. Learning in new Technological and Educational Contexts (pp. 181-204). Bern, Suiza: Peter Lang.

Berns, A., Palomo-Duarte, M., Dodero, J.M. e Isla-Montes, J.L. (2016). Motivation, students' needs and learning outcomes: a hybrid game-based app for enhanced language learning. SpringerPlus 5: 1305, pp. 1-23. doi:10.1186/s40064-016-2971-1.

Berns, A., Palomo-Duarte, M., Isla-Montes, J.L., Dodero, J.M. y Delatorre Moreno, P. (2017). Agenda colaborativa para el aprendizaje de idiomas: del papel al dispositivo móvil. RIED. Revista Iberoamericana de Educación a Distancia, 20 (2), pp. 119139. doi:10.5944/ried.20.2.17713.

Burston, J. (2013). Mobile-Assisted Language Learning: A selected annotated Bbbliography of implementation studies. Language Learning \& Technology, 17 (3), pp. 157-224. Recuperado de http://lit.msu.edu/issues/october2013/burston.pdf. 
Burston, J. (2014a). The reality of MALL project implementations: Still on the fringes. CALICO Journal, 31 (1), pp. 43-65. doi:10.11139/cj.31.1.103-125.

Burston, J. (2014b). MALL: the pedagogical challenges. Computer Assisted Language Learning, 27 (4), pp. 344-357. doi:10.1080/09588221.2014.914539.

Burston, J. (2015). Twenty years of MALL project implementation: A meta-analysis of learning outcomes. ReCALL, 27 (1), pp. 4-20. doi:10.1017/S0958344014000159.

Carpena, N., Cataldi, M., y Muñiz, G. (2012). En busca de nuevas metodologías y herramientas aplicables a la educación. Repensando nuestro rol docente en las aulas. Novos sistemas de produção. Recuperado de https://cutt.ly/VofTdPQ

Chinnery, G. (2006). Going to the MALL: Mobile Assisted Language Learning. Language Learning \& Technology, $10 \quad$ (1), pp. 9-16. Recuperado dehttp://llt.msu.edu/vol10num1/emerging/.

Duman, G., Orhon, G. y Gedik, N. (2015). Research trends in mobile assisted language learning from 2000 to 2012. ReCALL, $27 \quad$ (2), pp. 197-216. doi:10.1017/S0958344014000287.

Ermi, L. y Mäyrä, F. (2005). Player-centred game design: Experiences in using scenario study to inform mobile game design. Game Studies, 5 (1), pp. 1-10. Recuperado de http://www.gamestudies.org/0501/ermi_mayra/.

Engeström, Y. (1987). Learning by expanding: an activity-theoretical approach to developmental research. Helsinki: Orienta-KonsultitOy.

Faiella, F. y Ricciardi, M. (2015). Gamification and learning: A review of issues and research. Journal of E-Learning and Knowledge Society, 11 (3), pp. 13-21. doi: $10.20368 / 1971-8829 / 1072$.

Gikandi, J., Morrow, D. y Davis, N. (2011). Online formative assessment in higher education: A review of the literature. Computers \& Education, 57 (4), pp. 2333-2351. doi:10.1016/j.compedu.2011.06.004.

Godwin-Jones, R. (2011). Mobile apps for language learning. Language Learning \& Technology, $15 \quad$ (2), $\quad$ pp. 2-11. Recuperado de http://llt.msu.edu/issues/june2011/emerging.pdf.

Hasegawa, T., Koshino, M. y Ban, H. (2015). An English vocabulary learning support system for the learner's sustainable motivation. SpringerPlus, 4(99), pp.1-9. doi:10.1186/s40064-015-0792-2. 
Heil, C.R., Wu, J.S., Lee, J.L. y Schmidt, T. (2016). A review of mobile language learning applications: trends, challenges and applications, The EUROCALL Review, 24 (2), pp. 32-50. doi:10.4995/eurocall.2016.6402.

Kennedy, C. y Levy, M. (2009). Sustainability and computer-assisted language learning: factors for success in a context of change. Computer Assisted Language Learning, 22 (5), pp. 445-463. doi:10.1080/09588220903345218.

Kumar, B.A. y Mohite, P. J. (2018). Usability of mobile learning applications: a systematic literature review. Journal of Computers in Education, 5 (1), pp. 1-17. doi:10.1007/s40692-017-0093-6.

Kukulska-Hulme, A. y Shield, L. (2008). An overview of mobile assisted language learning: from content delivery to supported collaboration and interaction. ReCALL, 20 (3), pp. 271-289. doi: 10.1017/S0958344008000335.

Kukulska-Hulme, A. y Viberg, O. (2018). Mobile collaborative language learning: State of the art. British Journal of Educational Technology,49 (2), pp. 207-218. doi:10.1111/bjet.12580.

Lee, J.J. y Hammer, J. (2011). Gamification in Education: What, How, Why Bother? Academic Exchange Quarterly, 15 (2), pp. 146-151. Recuperado de shorturl.at/LT178.

Liu, I.F., Chen, M.C., Sun, Y.S., Wible, D. y Kuo, C.H. (2010). Extending the TAM model to explore the factors that aect intention to use an online learning community. Computers\&Education, 54 (2), pp. 600-610. doi:10.1016/j.compedu.2009.09.009.

Ministerio de Educación, Cultura y Deporte (2002). Marco Europeo de Referencia para las Lenguas: Aprendizaje, enseñanza, evaluación Recuperado de https://cutt.ly/3occJL2.

Monguillot Hernando, M., González Arévalo, C., Zurita Mon, C., Almirall Batet, L., y Guitert Catasús, M. (2015). Play the Game: gamification and healthy habits in physical education. Apunts. Educación Física y Deportes, 119, pp. 71-79. doi:10.5672/apunts.2014-0983.es.(2015/1).119.04

Nacke, L. E. y Deterding, Ch. S. (2017). The maturing of gamification research. Computers in Human Behaviour, 71, pp. 450-454. doi:10.1016/j.chb.2016.11.062.

Nelson, T. y Oliver, W. (1999). Murder on the Internet. CALICO Journal, 17 (1), pp. 101114. Recuperado de https://www.jstor.org/stable/24152617?seq=1.

Pérez López, J.I. (2019). Los (al menos) 7 pecados capitales en torno a la gamificación. Habilidad Motriz. Revista de ciencias de la actividad física y del deporte53, pp. 2-4. Recuperado dehttps://dialnet.unirioja.es/servlet/articulo?codigo=7110516. 
Pérez-Pueyo, A. y Hortigüela, A. (2020). ¿Y si toda la innovación no es positiva en Educación Física? Reflexiones y consideraciones prácticas. Retos, 37, pp. 579-587. Recuperado dehttps://recyt.fecyt.es/index.php/retos/article/view/74176/45761.

Palomo-Duarte, M., Berns, A., Cejas, A., Dodero, J.M. y Ruiz-Rube, I. (2016). Assessing foreign language learning through mobile game-based learning environments. Journal of Human Capital and Information Technology Professionals,7 (2), pp. 5367. doi:10.4018/IJHCITP.2016040104.

Palomo-Duarte, M., Berns, A., Cejas, A., Dodero, J.M., Caballero-Hernández, J.A. y RuizRube, I. (2018). A Community-Driven Mobile System to Support Foreign Language Learning. En V. Ahuja y Sh. Rathore (Eds.), Multidisciplinary Perspectives on Human Capital and Information Technology Professionals (pp. 95-115), IGI Global. doi: 10.4018/978-1-5225-5297-0.ch006.

Ryan, R.R. y Deci, E. L. (2000). Self-Determination Theory and the Facilitation of Intrinsic Motivation, Social Development, and Well-Being. American Psychologist55, (1),pp. 68-78. doi: 10.1037110003-066X.55.1.68.

Sein-Echaluce, M.L. Fidalgo-Blanco, A. y Alves, G. (2017). Technology behaviors in education innovation. Computers in Human Behavior, 72, pp. 596-598. doi:10.1016/j.chb.2016.11.049.

Sharples, M., Taylor, J. y Vavoula, G. (2016). A Theory of Learning for the Mobile Age. En, C. Haythornthwaite, R. Andrews, J. Fransman y E.M. Meyers (Eds.), The Sage Handbook of Elearning Research (pp.221-247), Washington DC, USA: Sage publications.

Subhash, S. y Cudney, E. A. (2018). Gamified learning in higher education: A systematic review of the literature. Computers in Human Behaviour87, pp. 192-206. doi:10.1016.j.chb.2018.05.028.

Taras, M. (2002). Using Assessment for Learning and Learning from Assessment. Assessment \& Evaluation in Higher Education, 27 (6), pp. 501-510. doi:10.1080/0260293022000020273.

Van Zundert, M.J., Sluijsmans, D. y van Merriënboer, J. (2010). Effective peer assessment processes: Research findings and future directions. Learning and Instruction, 20 (4), pp. 270-279.doi:10.1016/j.learninstruc.2009.08.004. 